\title{
Creating regular matrices of aligned silica nanohelices: theory and realization
}

\author{
Jie Gao, ${ }^{1}$ Sanaa Semlali, ${ }^{2}$ Julien Hunel, ${ }^{3}$ David Montero, ${ }^{4}$ Yann Battie, ${ }^{5}$ David Gonzalez-Rodri- \\ guez, ${ }^{5}$ Reiko Oda, ${ }^{1}$ Glenna L. Drisko, ${ }^{* 2}$ Emilie Pouget ${ }^{* 1}$
}

\begin{abstract}
${ }^{1}$ CNRS, Univ. Bordeaux, Bordeaux INP, Chimie et Biologie des Membranes et des Nanoobjets, UMR 5248, Allée St Hilaire, Bat B14, 33607 Pessac, France

${ }^{2}$ CNRS, Univ. Bordeaux, Institut de Chimie de la Matière Condensée de Bordeaux, UMR 5026, 87 av du Dr. Schweitzer, 33600 Pessac, France.

${ }^{3}$ CNRS, Univ. Bordeaux, Institut des Sciences Moléculaires, UMR 5255, 351 cours de la Libération, 33405 Talence, France.

4 Sorbonne Univ., Institut des Matériaux de Paris Centre (IMPC FR 2482), UFR de Chimie Campus Jussieu, 75252

Paris, France

${ }^{5}$ Laboratoire LCP-A2MC, Institut Jean Barriol, Université de Lorraine, 1, boulevard Arago, 57078 Metz cedex 3 ,

France.
\end{abstract}

KEYWORDS: Silica, Elongated nano-object, Helix, Evaporation-induced self-assembly, Dip-coating, Capillarity theory

\begin{abstract}
Here we exercise nanoscale control over the assembly of highly anisotropic silica helices using convective flow by using the physical properties occurring during evaporation-induced self-assembly. Organizing and patterning such chiral elongated objects over large surfaces in a controllable and reproducible fashion is challenging, but desirable to optimize the performance of biomimetic structures, nano-sensors (mechanical properties of helices) or optical materials (chiral objects have asymmetric interactions with light as absorption is different for left- and right-handed polarization). The coupling of evaporation forces and physicochemical solution properties induce specific helix alignment and the stick-slip phenomenon produces a periodical deposition of bands with controllable and regular spacing. Helix orientation, packing density and spacing can then be tuned. We observe the effect of polymer additives, silica helix concentration, and substrate withdrawal speed on the quality and the orientation of the helix deposition. Theoretical modeling based on capillary hydrodynamics is developed to describe the relationship between evaporative conditions and pitch distance, the band width of the stick region and the helix orientation.
\end{abstract}

1. Introduction

Organizing elongated nanometric objects into an ordered array can increase or generate properties unobserved when these objects are individually dispersed or randomly oriented. Material scientists have proposed various approaches to place and arrange complex anisotropic nanobuilding blocks. ${ }^{[1-3]}$ The self-templating process, ${ }^{[4,5]}$ organization via organic matrices, ${ }^{[6,7]}$ spraying, ${ }^{[8-10]}$ and evaporation based techniques are among the techniques available for guiding anisotropic object assembly. ${ }^{[1-16]}$ The formation of lyotropic liquid crystals directly in bulk or via these evaporation techniques represents an interesting tool. ${ }^{[1-19]}$ Where convective evaporation has generated beautifully organized patterned surfaces from inorganic anisotropic objects like nanowires and nanorods ${ }^{[12,15,20]}$ or chiral biological rod-like viruses, ${ }^{[21,22]}$ to the best of our knowledge, the organized assembly of synthetic chiral anisotropic objects has not yet been reported. Nanoscale helices behave differently than nanoparticles and rods due to the excellent coupling of sheer forces in liquid with helix particle motion. Such dynamics may help or destroy the organization, a question which we probe here.

Dip-coating does not require sophisticated and expensive equipment or a pre-treatment of substrates, and is achieved simply by withdrawing the substrates immersed in liquid with solutes or particles under controlled speed. Pre-etching, laser imprinted designs and complex chemical treatment processes can be added to increase the complexity, but are typically unnecessary. Dip-coating has 
been applied to many industrial fields, e.g. in the preparation of ceramics, ${ }^{[23]}$ films, and hybrid materials. ${ }^{[24]}$ Since an early study by Schunk, Hurd and Brinker 20 years ago, ${ }^{[25]}$ the mechanisms underlying this technique continue to intrigue researchers. During evaporation, complex interactions are present between the solutes, solution and the surrounding environment at the interface, as well as in the bulk, affected by various conditions including chamber temperature, humidity, solution concentration, surface tension, viscosity and withdrawal speed. When this occurs in the presence of particles, they are concentrated at the drying line by convective transport driven by evaporation, and then tightly packed due to interparticle capillary forces. ${ }^{[26-28]}$ Not only do nanoparticles become pinned to the surface by the meniscus, but the meniscus becomes pinned to the surface by nanoparticles. Substrate withdrawal stretches the pinned meniscus and eventually unpins it, resulting in regular and compact particle-loaded lines separated by zones of poorly covered surfaces. This process is called the stick-slip phenomenon and can be used to produce regular and controllably spaced linear arrays of particles. ${ }^{[29]}$

Spheres or small aspect ratio particles can roll more easily than non-round objects with large aspect ratio and thus it is relatively easy to process them into dense assemblies. The equilibrium between the particle-substrate or particle-particle electrostatic interaction repulsions and van der Waals interaction forces can prevent tight particle packing. The movement of particle suspensions with more complex shapes are plagued by more complex interparticle interactions and higher drag forces along the substrate, increasing the difficulty of obtaining well-organized monoor multilayered structures. Once evaporation thins the meniscus to the thickness of the nano-object diameter, the objects can no longer diffuse or freely rotate and can only slide closer to or away from nearby objects. Also, the convective vs shear gradient forces can induce nanoparticle orientations parallel and perpendicular to the contact line (the meniscus). ${ }^{[22,23,29,30]}$ Finally, the physical-chemical properties of the solution (surface tension, viscosity, $\mathrm{pH}$, etc.), as well as the withdrawal speed, can all play a crucial role in surface patterning. These forces can control the formation of nematic-like phases via entropy gain in the concentrated and confined zones, as shown with carbon nanotubes, ${ }^{\left[{ }^{13}\right]} \mathrm{ZnO}$ nanorods, ${ }^{[21]}$ gold nanorods ${ }^{[12]}$ or biological filaments. ${ }^{[22,23]}$ Because the helical particles present different sheer forces coupling in liquid than more common rods, their assembly should be altered but has never been studied because of the lack of model systems at the nanoscale. However, we expect these assemblies of helices to open up possible applications in chiroptical devices.

In this article, we propose a complete study of the organization of highly anisotropic silica nanohelices over large surfaces, coupled with analytical hydrodynamic theory of the observed phenomena. We produce stable colloidal solutions of nanohelices in a mixture of ethanol and isopropanol and study their organization by evaporation during dip-coating on silicon substrates. This convective assembly of silica helices is studied by examining the effect of polymer additives, withdrawal speed and silica helix concentration. These different parameters are studied and optimized to accurately control the nanohelix deposition density, macroscopic organization and nanometric nematic order alignment. The obtained morphologies of organized helices are interpreted using theoretical modeling based on capillary hydrodynamics, giving insight into the physicochemical processes at work during the complex and constantly evolving environment of drying films.

\section{Results and discussions}

The sol-gel transcription of self-assembled $\mathrm{C}_{2} \mathrm{H}_{4}-\mathbf{1 , 2}$ $\left(\left(\mathrm{CH}_{3}\right)_{2 n}+\mathrm{C}_{16} \mathrm{H}_{33}\right)_{2}$ with a tartrate counterion yields nanometric silica helices with controlled morphology $(21 \mathrm{~nm}$ in width and a pitch of $92 \mathrm{~nm}$ ) and handedness (Figure 1). ${ }^{\text {[32- }}$ 34] These helices are initially long and entangled. Tip sonicating their suspension after transcription can fragment them into disentangled strands, micrometers in length. ${ }^{[35]}$ These strands form stable and homogeneously dispersed colloidal suspensions in a range of solvents, such as the mixture of ethanol and isopropanol used in the present study.

Evaporation-induced self-assembly is affected by the solution properties (viscosity, colloidal stability, colloidal concentration, etc.) and the evaporation rate. In this study, polymer additives, which modify the surface tension of the solution, the viscosity, the evaporation rate and also the steric and/or electrostatic particle-particle interactions, impact the deposition of the silica nanohelices. First, the suspensions (Isoelectric point in water $\sim 4.3$, see zetapotential measurement in Supporting Information 1) of the nanohelices were dip-coated onto a silicon substrate, with and without polymer additives (Figure 1). Anionic polyacrylic acid (PAA) and cationic polyethyleneimine (PEI) are the two linear polyelectrolytes used in this study, chosen as they present opposite charges at neutral $\mathrm{pH}$. Although it is difficult to probe the surface charge in alcoholic suspensions, the impact of the presence or absence of charged polymers on the aggregation of the colloidal silica helices in suspensions is visible using the electron microscopy images in Figure 1 and their different scattering properties (Supporting Information 2). The homogeneity of the surface coating is directly influenced by the presence or absence of the polymer in the suspension: in the absence of polyelectrolyte (Figure $1 \mathrm{~b}$ ), the deposition conditions applied here led to a sparse, disordered and inhomogeneous helix coverage. The addition of cationic PEI increases the surface density of silica nanohelices, compared to suspensions with no polyelectrolyte, probably due to a lower evaporation rate, allowing the more efficient pinning of the helices on the surface. However, the helices are still inhomogeneously distributed and randomly oriented (Figure 1c), likely due to the attractive interaction between cationic PEI and negatively charged silica surface. This Coulombic attraction leads to their aggregation and PEI crosslinking between nanohelices. On the contrary, in the presence of the anionic PAA, the helices are well oriented parallel to each other (Figure 1d). Perhaps the co-suspension of negatively charged PAA and negatively charged nanohelices is 
more stable with higher helix mobility. Additionally, PAA decreases the evaporation rate, giving the nanohelices time to align. PAA incorporation in the solution allows helices to be aligned vertically or horizontally, with controlled interband spacing.

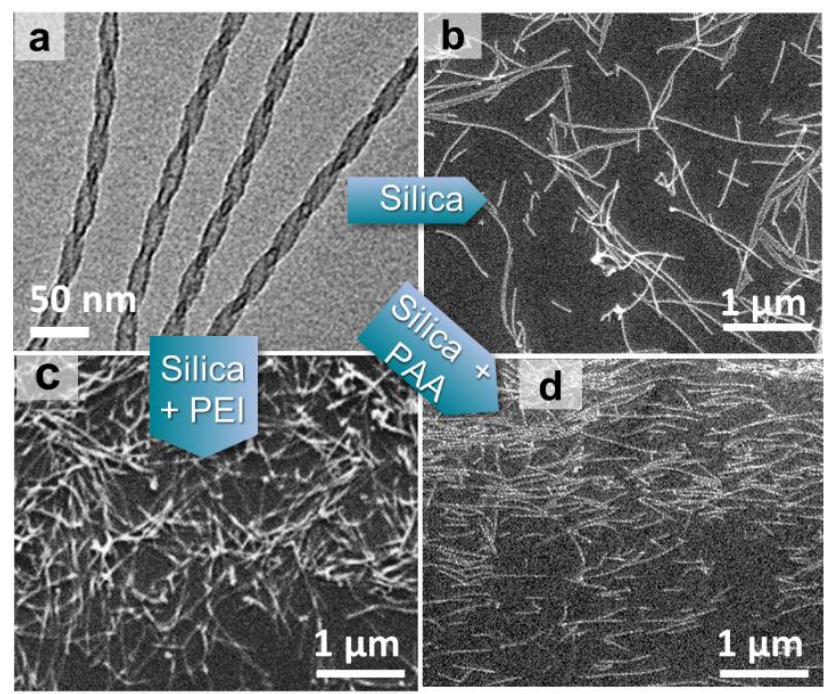

Figure 1. Effect of surface functionalization on helix assembly. (a) Transmission electron microscopy (TEM) image of individual silica helices. (b-d) Scanning electron microscope (SEM) images for the assemblies of silica helices with (b) no polyelectrolyte, (c) PEI and (d) PAA. The withdrawal speed is $5 \mu \mathrm{m} / \mathrm{s}$ and the helix concentration is $0.4 \mathrm{mg} / \mathrm{mL}$.

\subsection{Withdrawal speed: evidence of two evaporative regimes}

Numerous reports show that during dip-coating the withdrawal speed is closely related to the drag forces and the evaporation conditions strongly affect film thickness. ${ }^{[36,37]}$ We studied the effect of the withdrawal speed between 2.5 and $30,000 \mu \mathrm{m} / \mathrm{s}$ on the deposited film formed by the silica helices (Figure 2). The deposition density is defined as the percentage of the surface covered by helices divided by the total surface. In the presence of multilayers of helices in some regions, this percentage is multiplied by the number of layers (estimated by AFM characterization), giving numbers that can exceed 10o\% deposition density (See detailed image treatment in Supporting Information 3).

As observed in Figure 2, the density of helices covering the surface is high at the slowest withdrawal rates and decreases with increasing withdrawal speed until poor coverage occurs at withdrawal rates between 100-1000 $\mu \mathrm{m} / \mathrm{s}$. This density remains low until it increases again at the withdrawal speed of $\sim 10000 \mu \mathrm{m} / \mathrm{s}$, although it does not reach the high values observed at the slowest speeds. From the previous studies, these two regions of higher particle deposition density are explained by the presence of two physical regimes, governed by evaporative transport and by advective transport, respectively. ${ }^{[36]}$ The regime at slow withdrawal rate corresponds to the evaporative transport regime (in blue in Figure 2), where film thickness decreases with increasing withdrawal speed. The regime at fast withdrawal rate corresponds to the advection regime (in violet in Figure 2), where the film thickness increases with withdrawal speed. In both regimes, well-organized bands appear on the substrate: horizontal ones in the evaporative transport regime due to the stick-slip phenomenon (periodic helix-rich bands, the stick regions, alternate with helix-poor areas, the slip regions); vertical ones in the advection regime (Figure 3). The orientation of the helices in the bands is analyzed by Image-J as shown in the color scheme in Figure 3g. Light blue indicates an orientation of $\mathrm{o}^{\circ}$, the horizontal direction, where red indicates $\pm 90^{\circ}$, the vertical direction, from which the order parameter $S$ can be extracted (see Experimental section and Supporting Information 4). A high order parameter $S$ between o.850.95 in the horizontal orientation happens for the stick regions for the evaporative transport regime. The SEM image in Figure $3 \mathrm{~b}$ details the border of a slip and stick region, confirming the horizontal orientation of the helices in the stick bands. At an intermediate speed (30-1000 $\mu \mathrm{m} / \mathrm{s}$ ), the order parameter is low (0.2-0.3), indicating a mostly random orientation (Figure $2 \mathrm{c}$ ) with a slight alignment preference for $90^{\circ}$, suggesting that helices are subjected to higher shear, but without enough time to fully align. At a withdrawal speed ranging from 5000 to 30000 $\mu \mathrm{m} / \mathrm{s}$, the deposition density increases with a preferential alignment of $90^{\circ}$ (parallel to the withdrawal shear) with an average order parameter $S$ of 0.75 (Figures $2 \mathrm{~d}$ and $2 \mathrm{e}$ ). 

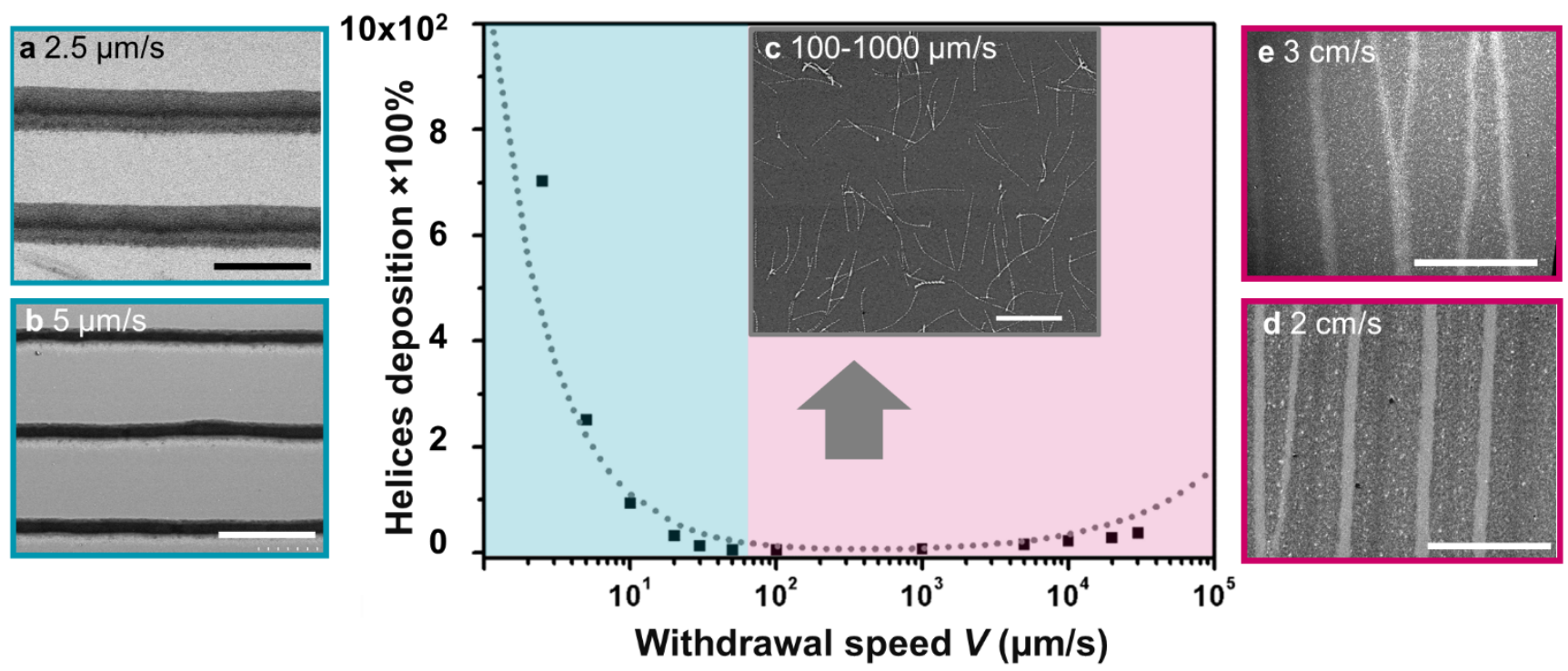

Figure 2. Helix deposition vs withdrawal speed. (a-e) SEM images at various withdrawal speeds between 2.5-30 ooo $\mu \mathrm{m} / \mathrm{s}$. The $\mathrm{SiO}_{2}: \mathrm{PAA}$ ratio is 1:10, the silica concentration is $0.4 \mathrm{mg} / \mathrm{mL}$. The dotted line indicates the theoretically predicted dependence of particle deposition density $\left(\rho_{P}\right)$ as a function of the withdrawal rate. Scale bars $(\mathbf{a}, \mathbf{b}, \mathbf{d}$ and $\mathbf{e})$ represent 100 $\mu \mathrm{m}$, and $(\mathbf{c}) 1 \mu \mathrm{m}$.

The density data vs withdrawal rate in Figure 2 can be described by a theoretical model. The two competing particle transport mechanisms, can be quantified as follows:

$$
\begin{aligned}
& Q_{a} \approx c h V \\
& Q_{e} \approx c l_{c} V_{e}
\end{aligned}
$$

Here $Q_{a}$ is the nanohelix transport rate towards the liquid meniscus due to particle advection into the Landau-Levich film driven by substrate motion. This advective transport rate increases with the withdrawal speed $V$ and is proportional to the particle concentration $c$ and to the thickness of the Landau-Levich film $h . Q_{e}$ is the nanohelix transport rate towards the meniscus due to evaporation, and $V_{e}$ is the evaporation speed. The typical length over which evaporation contributes to particle deposition scales with the capillary length $l_{c}$, the typical size of the meniscus, as this will be the typical size of the flow recirculation region. The number of particles deposited per unit surface of the plate, which is denoted $\rho_{P}$, can be estimated by:

$\rho_{P}=\frac{Q_{a}+Q_{e}}{V} \approx c l_{c} C a^{2 / 3}+c l_{c} \frac{\mathrm{V}_{e}}{V}=c l_{c}\left[\left(\frac{\mu V}{\gamma}\right)^{2 / 3}+\frac{V_{e}}{V}\right]$

where $\rho_{P}$ is the particle deposition density, $Q_{a}$ is the advective transport rate, $Q_{e}$ is the evaporative transport rate and $V$ is the withdrawal rate of the substrate. Variable $c$ is the particle concentration in solution, $l_{c}$ is the capillary length or typical size of the meniscus, and $C_{a}$ is the capillary number. $C_{a}=\mu V / \gamma$; where $\mu \approx 10^{-3}$ Pa.s is the dynamic viscosity of ethanol and $\gamma \approx 20 \mathrm{mN} / \mathrm{m}^{1}$ is the surface tension coefficient of ethanol. With a density $\rho \approx 800 \mathrm{~kg} / \mathrm{m}^{3}$, the capillary length of ethanol at $25^{\circ} \mathrm{C}$ is estimated to be $l_{c} \approx 1.6 \mathrm{~mm}$. The evaporation rate of ethanol at $25^{\circ} \mathrm{C}$ is estimated from published data ${ }^{[38]}$ to be the order of $V_{e}=0.2 \mu \mathrm{m} / \mathrm{s}$. This model reproduces the experimental observation in Figure
2. The fit, plotted as a dashed line, has the predicted $\rho_{P}$ scaled by a proportionality constant to fit the data. At low withdrawal speeds, evaporation at the meniscus creates the dominant flux of suspended particles. For $V<50 \mu \mathrm{m} / \mathrm{s}$, particle deposition is inversely proportional to $V$, as it is dominated by liquid evaporation. As the plate is withdrawn faster, the plate spends less time in contact with the meniscus, where evaporation-driven deposition occurs. Thus, particle deposition density decreases dramatically from $700 \%$ at a withdrawal speed of $2.5 \mu \mathrm{m} / \mathrm{s}$ to $13 \%$ at a speed of $30 \mu \mathrm{m} / \mathrm{s}$. For $V>1000 \mu \mathrm{m} / \mathrm{s}$, the dominant deposition mechanism becomes advection, where particle transport is driven by the motion of the substrate. Advection increases with the thickness of the Landau-Levich film, $\rho_{P} \approx V^{2 / 3}$. In the intermediate regime, $50<V<1000$ $\mu \mathrm{m} / \mathrm{s}$, both evaporation-induced transport and advection are weak, and particle deposition density reaches its minimum.

As described in previous studies, ${ }^{[39,40]}$ there is a transition occurring from evaporative to advective transport which corresponds to the condition $Q_{e}=Q_{a}$. By balancing Equations (1) and (2) and by considering the current experimental conditions, we obtain a critical speed: $V_{\text {crit }}=V_{e}{ }^{2 / 5} \approx 300 \mu \mathrm{m} / \mathrm{s}$. This result is consistent with the recorded data presented in Figure 2.

\subsection{Evaporative transport regime: controlled stick- slip phenomenon}

At low withdrawal speeds, in the evaporative transport regime, a clear stick-slip phenomenon appears where periodic helix-rich bands, the stick regions, alternate with helix-poor areas, the slip regions (Figures $2 a$ and $2 b$ ). Because of the low withdrawal speed used here, the meniscus becomes stretched as the drying line slowly moves farther 
a
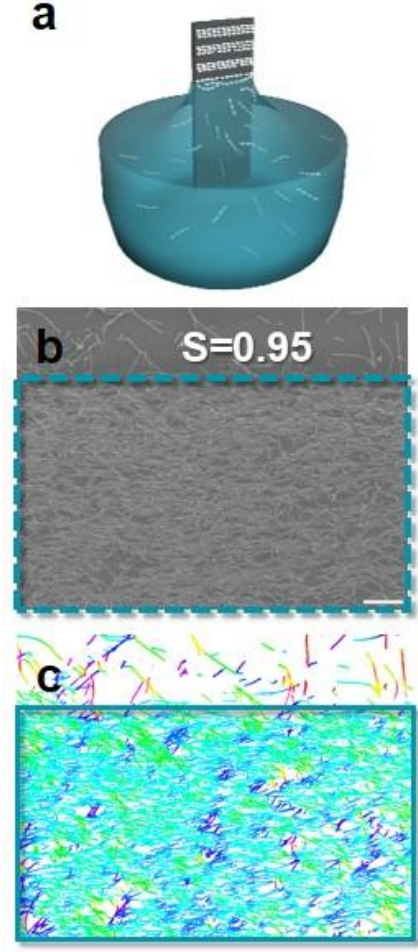

d
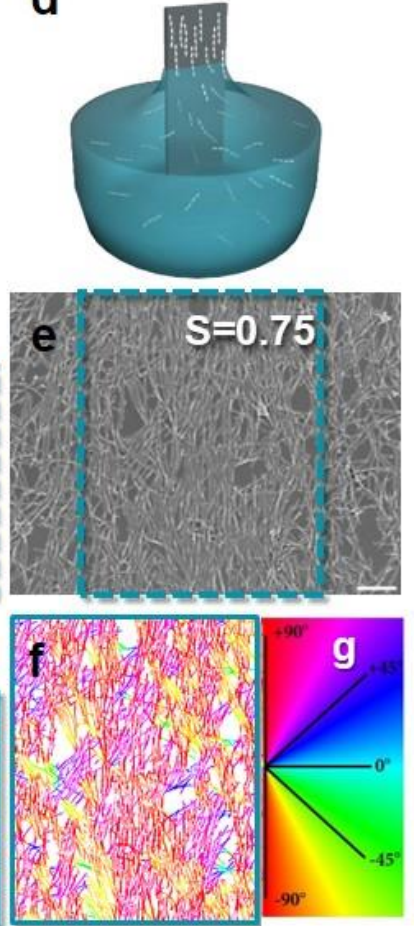

Figure 3. Controlling orientation through withdrawal speed. (a-c) The horizontal and (d-f) the vertical alignment $(\mathbf{a}, \mathbf{d})$ scheme, (b,e) SEM image, and (c,f) orientation analysis. The colloidal solution is silica:PAA=1:10, the silica concentration is $0.4 \mathrm{mg} / \mathrm{mL}$ and the withdrawal speed is either (a-c) $10 \mu \mathrm{m} / \mathrm{s}$ or $(\mathbf{d}-\mathbf{f}) 3000 \mu \mathrm{m} / \mathrm{s}$. (g) is the legend for the colors shown in (c) and (f), with respect to various angles. Scale bars represent $1 \mu \mathrm{m}$.

away from the bulk solution as the substrate is withdrawn. As long as the meniscus is pinned to the drying line, more helices can accumulate in the drying zone, leading to a higher concentration of particles than that of the bulk solution. At some point, the meniscus is so stretched that it overcomes the particle pinning forces and quickly jumps to its resting position, where it is pinned again by a fresh set of deposited particles. This periodic pinning and break-up of the meniscus is well-known in the evaporative transport regime as the stick-slip phenomenon. ${ }^{41}$ The fact that nanohelices accumulate at the meniscus instead of entering into the wetting film crucially depends on the ratio between particle size and film thickness, as has been demonstrated for spherical particles through numerical simulation. ${ }^{[42]}$ For nanohelices, we must additionally consider that their trajectories follow what is called a Jeffery orbit ${ }^{[43]}$ as they are transported through solution. The Jeffery orbit has been first studied for the motion of rigid elliptical particles in shear flow, which rotate periodically. At slow withdrawal rates, the wetting film is too thin for helices to enter via a Jeffery orbit and so the helices become trapped in the meniscus. Helix deposition is then driven by evaporative transport in this meniscus, by a similar mechanism as that described for spherical particles. ${ }^{[44,45]}$ In the presence of helices, the meniscus becomes pinned (Figure $4 \mathrm{~b}$ ). Starting from the initial meniscus position (Figure 4a), the distance between the pinning point and the substrate's surface is denoted $h_{p}$. This distance $h_{p}$ increases with time, as a result of helix deposition. The slower the withdrawal rate, the more helices are deposited and the larger is the helix stick region. The motion of the substrate as it is withdrawn stretches the meniscus. Due to capillary-induced curvature, the meniscus eventually touches the surface of the substrate and breaks up (Figure 4c). The two resulting menisci rapidly retract to recover their static shape with a contact angle $\theta_{c}$ (Figure $4 \mathrm{~d}$ ). The upper and lower meniscus continue to retract over time due to evaporation. The motion of the receding contact line during evaporation will tend to align the nanohelices in the direction of the horizontal band, showing nematic-like phase organization.

In order to further control the patterning of surfaces, the effect of the mass ratio of $\mathrm{SiO}_{2}: \mathrm{PAA}$ on the helix assembly and stick-slip morphologies was investigated from 1:o to 1:20 for a withdrawal speed of $5 \mu \mathrm{m} / \mathrm{s}$ and a silica nanohelix concentration of $0.4 \mathrm{mg} / \mathrm{mL}$ (Figure 5). Increasing the PAA concentration enhances the width of the stick region and

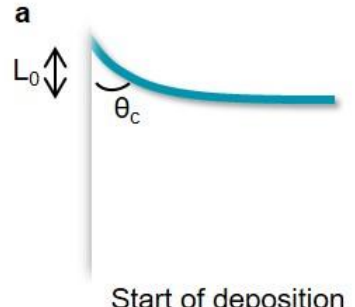

Start of deposition b

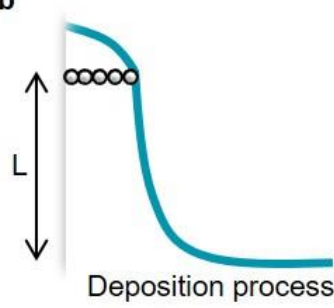

C

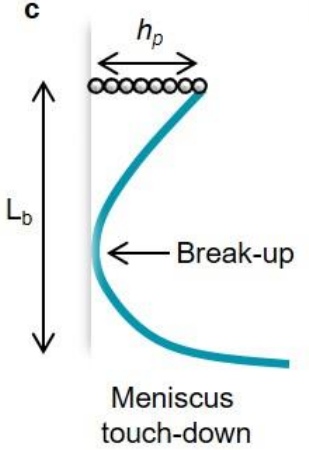

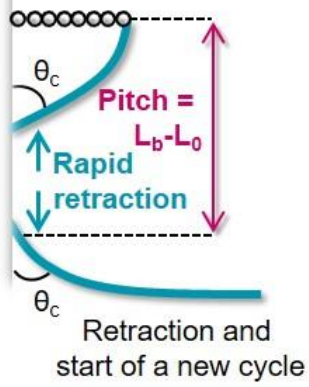

Figure 4. Conceptualization of the horizontal strip formation. (a) The start of the stick zone deposition. (b) Intermediate stage. (c) The meniscus touches the substrate, breaks-up and ends deposition in the current stick zone. (d) After meniscus breakup, rapid retraction occurs, which contributes to the pitch between stick zones. 
the helix concentration in these zones, because the helix transport rate increases with concentration for a given evaporation rate. We also note that higher concentration of PAA may additionally lower the evaporation rate. The orientation of the helices inside the stick regions is also influenced by the polymer additive: $S$ increases from 0.02 in the absence of PAA to a maximum of 0.86 for a mass ratio of $\mathrm{SiO}_{2}: \mathrm{PAA}$ of 1:10, confirming the importance of the presence of the negatively charged polymer to the free rotational motion of the helices.
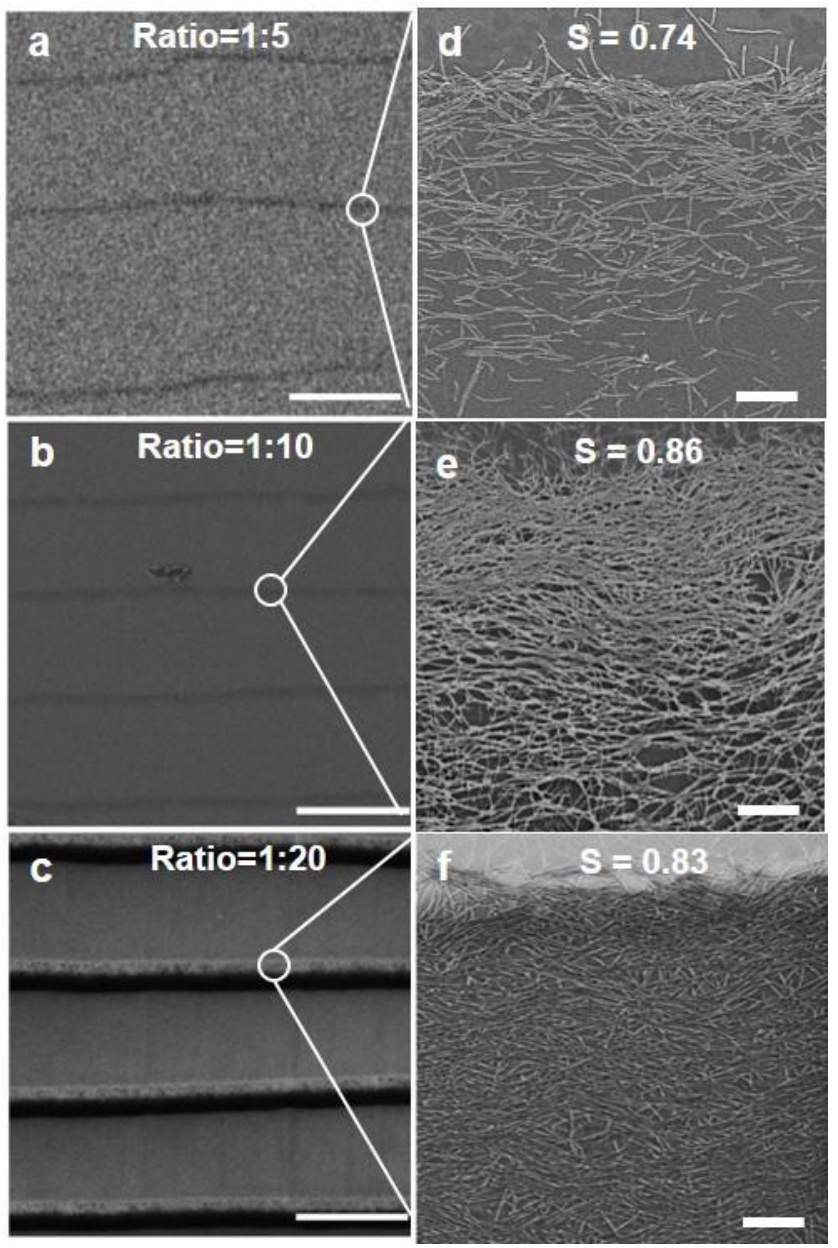

Figure 5 . Dependence of order on $\mathrm{SiO}_{2}: \mathrm{PAA}$ ratio. SEM images of the dip-coated samples with $\mathrm{SiO}_{2}$ :PAA ratio of (a) 1:5, (b) 1:10 and (c) 1:20. The images (d-f) show higher magnification of the stick regions of (a-c). The withdrawal speed was 5 $\mu \mathrm{m} / \mathrm{s}$, the helix concentration was $0.4 \mathrm{mg} / \mathrm{mL}$. Scale bars represent $100 \mu \mathrm{m}$ for (a-c) and $1 \mu \mathrm{m}$ for (d-f).

The stick-slip phenomenon gives controlled surface patterning and can be tuned via the silica concentration of the nanohelix suspensions. Figure 6 shows the evolution of this patterning from 0.1 to $1 \mathrm{mg} / \mathrm{mL}$ silica helix solutions with a ratio $\mathrm{SiO}_{2}: \mathrm{PAA}$ of 1:10 and a withdrawal speed of $5 \mu \mathrm{m} / \mathrm{s}$. The samples observed by polarized optical microscopy in the reflection mode show a black background corresponding to the slip regions and light lines corresponding to the stick regions.

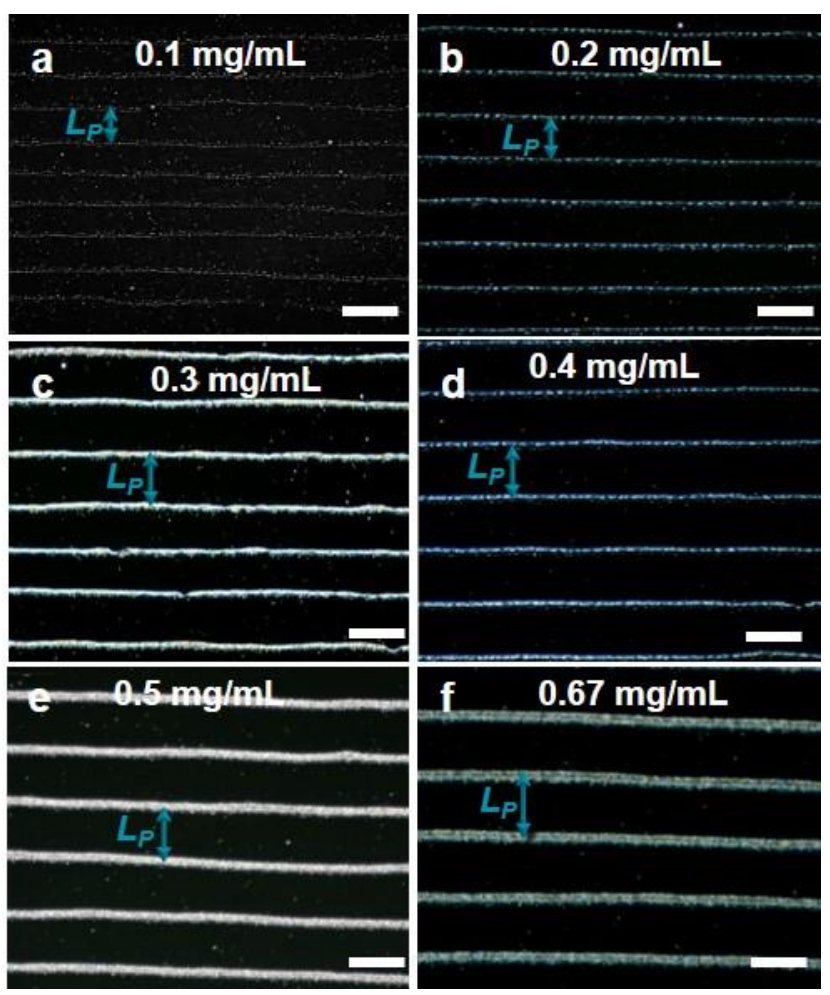

Figure 6. Dependence of pitch on silica concentration. (af) Reflection mode polarized optical microscopy images of substrates prepared using a silica concentration ranging from 0.1 to $1 \mathrm{mg} / \mathrm{mL}$. The $\mathrm{SiO}_{2}$ :PAA ratio used was $1: 10$, the withdrawal speed was $10 \mu \mathrm{m} / \mathrm{s}$. Scale bars represent $100 \mu \mathrm{m}$.

As plotted in Figure $7 \mathrm{~b}$, the pitch between two stick regions increases with increasing silica concentration. Due to the higher helix concentration in solution, more helices are deposited during drying. Due to the more significant quantity of helices at the drying line, the meniscus is pinned at a larger distance from the substrate $h_{p}$, resulting in a bigger jump to its static equilibrium position upon unpinning (Figure 4). Theoretical calculations of pitch $\left(L_{p}\right)$ as a function of helix concentration have been performed; details of the theoretical modeling can be found in the Experimental section. The theoretical model shows a good fit to the experimental data (Figure 7). The pitch increases with concentration rapidly at low concentrations and more slowly at higher concentrations. In parallel, atomic force microscopy was used to accurately measure the height of the nanohelix stacking (width) and the thickness of the stick regions (Figure $7 \mathrm{c}$ ). The width and thickness of the stick region increase with silica concentration. Moreover, experimental data in Figure $7 \mathrm{~b}$ shows that the width is proportional to the thickness. This suggests that the shape of the helix deposition may be determined by the evaporation of the remnant solution after the stick-slip occurs. The evaporating drop of liquid has a constant contact angle that is independent of particle concentration. The tangent of this contact angle may thus set a constant ratio between the height and the thickness of the deposit. 

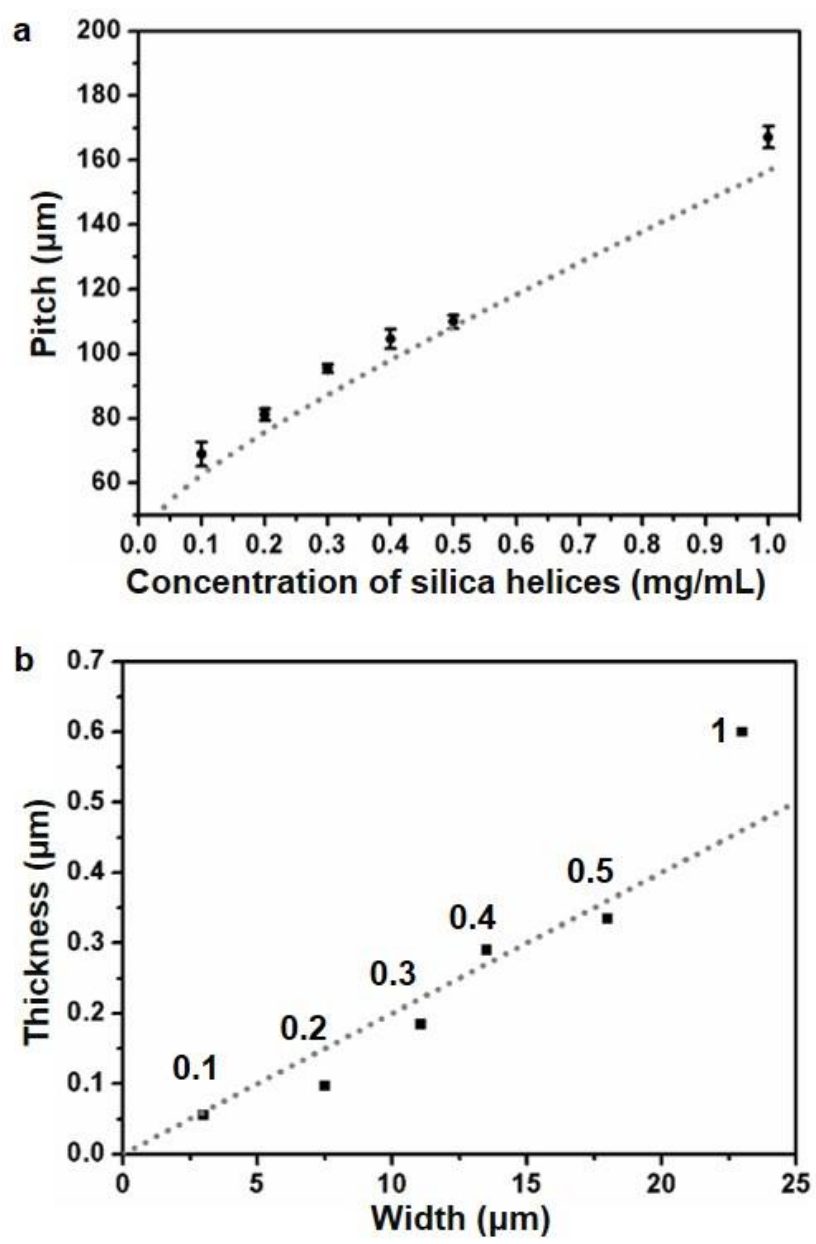

c

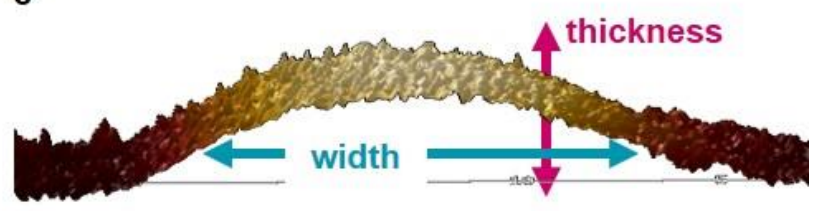

Figure 7. Theoretical modelling of experimental stick-slip variations. (a) Stick-slip pitch vs helix concentration, measured from the optical microscopy images in Figure 6. (b) Stick thickness vs stick width measured using AFM for all the studied concentrations. (c) Side view of AFM image one stick line for a silica concentration of $0.4 \mathrm{mg} / \mathrm{mL}$, showing the definition of width and thickness of the stick regions. In (a) and (b), the dotted line shows the theoretical predictions of the evaporative transport model.

\subsection{Advection regime: the unexpected vertical bands}

In the advection regime, no particle accumulation due to evaporation occurs; the concentration of helices in the liquid film is equivalent to that of the bulk solution. Moreover, in the advection regime, the meniscus is thicker than the length of the helices. Thus, helices are aspirated into the meniscus via advection, spinning in a Jeffery orbit (Supporting Information 7). Once inside the drying film, advection velocity dominates over rotational diffusion and so the helices will tend to align with the flow, ${ }^{[46]}$ referred to as shear flow. Although this explains the vertical alignment of the nanohelices (Figure $3 \mathrm{~d}-\mathrm{f}$ ), it does not explain their organization into bands of nanohelices (Figure $2 \mathrm{~d}, \mathrm{e}$ ). Few examples are known of such bands forming in the draining regime because of shear force. ${ }^{[47,48]}$ Two factors seem to be the origin of the band formation: the elongated shape of the nanohelices and the presence of PAA. As shown in the Supporting Information 5, if the same experiment is performed with spherical silica nanoparticles of similar diameter and in the presence PAA, no bands form, but a homogeneous deposition is observed. And if the same experiment is performed with silica helices, but without the presence of PAA, helices are randomly deposited without any obvious organization. Moreover, in a film containing the helices and PAA, a hexagonal organization is observed if the substrate dries horizontally (Supporting Information 6), showing that helices still align relative to each other. This confirms that the shear forces induced by gravity driven draining alone are not enough to cause the alignment of the helices, but the presence of the polymer acting as a lubricant is required. Furthermore, the vertical band formation seems to be a consequence of these shear forces during the final evaporation stage after substrate withdrawal, as the bands only appear when the sample is dried vertically. Such vertical band formation is reminiscent of experiments performed by Huang and collaborators, [49] where stripes of nanoparticles formed perpendicular to a dewetting front. The gravity effect in our experiments appears necessary to drive the propagation of the dewetting front. The mechanism leading to the formation of bands perpendicular to a drying front remains unknown, although a relation to a fingering instability is possible. ${ }^{\left[{ }^{0}, 51\right]}$

\section{Conclusion}

In this study, we have shown that we can orientate these chiral elongated nano-objects in a chosen direction and organize them in periodic structures in arrays of adjustable density. Indeed, large surfaces can be covered with organized silica nanohelices of controlled patterns, deposition density and object orientation. The effect of various parameters (polymer additives, nano-object concentration and withdrawal speed) on the organization was investigated to understand the formation mechanism. Thick bands can be obtained perpendicular to the withdrawal direction with a controlled periodicity and a nematic alignment of nanohelices within each band when working in the evaporative transport mode (slow withdrawal). Less dense bands can be obtained parallel to the withdrawal direction in the advection mode (rapid withdrawal), also showing a good nematic order. The reason for orientation can be explained by theoretical considerations based on fluid mechanics, where capillary contraction orients the helices parallel to the contact line at low velocity, and shear forces orient the helices perpendicularly at high velocities. Using a theoretical model based on capillary hydrodynamics, we were able to predict the observed pitch width and distance. Thus, the model predicts the morphologies that should be obtained by varying the different experimental parameters. It would be particularly interesting to then extend this work to objects with unique chiroptical properties, such as 
gold-based helices. ${ }^{[55,56]}$ The organization of these elongated and chiral nano-objects is of great interest for applications that are presently being developed in our laboratory, such as nano-sensors and model systems for cell differentiation to generate new biological tissues.

\section{Experimental Section}

Silica nanohelix colloidal solutions: The solutions were prepared following three steps: synthesis of the organic template, silica transcription and helix dispersion; each of which has been described by Okazaki, et al. ${ }^{[35]}$

Step 1: the synthesis of organic surfactant template $\mathrm{C}_{2} \mathrm{H}_{4}$ 1,2- $\left(\left(\mathrm{CH}_{3}\right)_{2} \mathrm{~N}^{+} \mathrm{C}_{16} \mathrm{H}_{33}\right)_{2}$ with a tartrate counterion, also named 16-2-16 Gemini L-tartrate, was optimized in a previous work. ${ }^{[32]}$ First, 16-2-16 Gemini bromide was obtained from the reaction of $\mathrm{N}, \mathrm{N}, \mathrm{N}^{\prime}, \mathrm{N}^{\prime}$-tetramethylethylenediamine with 1-bromohexadecane. Then, silver acetate was used to replace the bromide in 16-2-16 Gemini bromide, giving the product 16-2-16 Gemini acetate. Finally, L-tartaric acid was chosen for the ion exchange of the acetate, to give the 16-2-16 Gemini L-tartrate. The complex self-assembles in water to form helices. $1 \mathrm{mM} \mathrm{16-2-16} \mathrm{L-tartrate}$ aqueous solution was heated to $60^{\circ} \mathrm{C}$ for $15 \mathrm{~min}$, then the solution was cooled to $20^{\circ} \mathrm{C}$ for $2 \mathrm{~h}$.

Step 2: TEOS (50o $\mu \mathrm{L})$ was added to $10 \mathrm{~mL}$ of o.1 $\mathrm{mM}$ aqueous solution of L-tartaric acid ( $\mathrm{pH}$ 3.8) and prehydrolyzed at $20{ }^{\circ} \mathrm{C}$ by stirring on a roller-mixer for $7 \mathrm{~h}$. Then equal volumes of prehydrolyzed TEOS and organic gels containing the helices are mixed (typically, $4 \mathrm{~mL}$ of each) and stirred at $20^{\circ} \mathrm{C}$ with a roller-mixer overnight. The excess TEOS and organic template were washed away by isopropanol and ethanol using a centrifuge.

Step 3: A high intensity ultrasonic processor (Vibra cell 75186) equipped with a $2 \mathrm{~mm}$ microtip with variable power was used (maximum power, $130 \mathrm{OW}$ ). A $20 \mathrm{kHz}$ pulse mode was used for the dispersion and fragmentation of silica nanohelices. $2 \mathrm{mg}$ of silica nanohelices were mixed with $2 \mathrm{~mL}$ of a 1:1 mixture of ethanol:isopropanol and sonicated for 15 min with pulses of $1 \mathrm{~s}$, separated by $1 \mathrm{~s}$ pauses. Samples were cooled in an ice bath during the sonication process in order to avoid solvent evaporation and an increase of the sample temperature, which could influence the fragmentation process.

Finally, PEI and PAA were dissolved in ethanol to a concentration of $20 \mathrm{mg} / \mathrm{mL}$. The polymer solutions and silica suspensions were mixed and then sonicated for $10 \mathrm{~min}$ in an ultrasound bath to produce homogenous suspensions. Both PEI and PAA are linear polymers: PEI was purchased from Polysciences, Inc. with a molecular weight of 100,00og/mol. PAA was purchased from Sigma Aldrich with a molecular weight of $450,000 \mathrm{~g} / \mathrm{mol}$.

Dip-coating: Dip coating experiments were performed on an ACEdip 2.o dip-coater from SolGelWay. The withdrawal speed, and the chamber temperature were controlled using the software ACEdip 2.0 SOLGELWAY. All experiments were conducted using a chamber temperature of $25^{\circ} \mathrm{C}$. Boron-doped prime $\mathrm{CZ}$ silicon wafers with (100) orientation were obtained from Sil'tronix. The wafers were cleaned, rinsed with absolute ethanol and wiped dry with a Kimwipe paper, before use as the substrate for helix self-assembly.

Calculation of the order parameter, $S$ : The distribution of the acute angles $(\theta)$ between the principal axis and each nanohelix orientation was determined using the binary images extracted from the SEM images (Supporting Information 5). The Orientation-J pluggin developed for ImageJ corresponding to the structure tensor in a local neighborhood was used. ${ }^{[52,53]}$ A 2 D nematic order parameter $S$ can be calculated from this angular distribution:

$$
S=\left\langle 2 \cos ^{2} \theta-1\right\rangle
$$

where $\theta$ is the angle between the helices and the primary orientation. $S=0$ corresponds to a random distribution and $S=1$ corresponds to a perfectly parallel alignment.

Scanning electron microscopy (SEM): Before the preparation of the SEM samples, the slides are treated with oxygen plasma for $6 \mathrm{~min}$ in order to evaporate the polymer layers embedding the aligned helices. To show the initial distribution of PAA relative to silica helices, typical SEM images of samples with and without this plasma treatment are shown in SI-8. Then, the silicon slides are fixed with double face carbon tape on a classical SEM stub and covered with a $\mathrm{Au} / \mathrm{Pt}$ layer a few $\mathrm{nm}$ in thickness, deposited by plasma sputtering. Two FESEM have been used for the observation: JSM 670oF JEOL, and SEM-FEG Hitachi SU-70.

Atomic Force Microscopy (AFM): AFM analysis of the topology of the dip-coated films was performed on a Dimension Icon ScanAsyst from Bruker. The thickness and width of the stick region was measured precisely using an AFM with a silicon nitride probe. The parameters for measurement were a PeakForce frequency of $2 \mathrm{kHz}$ and a scan tip rate of $0.125 \mathrm{~Hz}$. The probe used has a tip radius of $5.0 \mathrm{~nm}$ and a constant stiffness of $0.4 \mathrm{~N} / \mathrm{m}$.

Polarized Optical Microscopy: Silicon wafers with a deposited pattern were observed in the reflection mode. A Nikon DXM 1200 camera and Lucia G software with an objective magnification of $\times 10$ were used to collect optical microscopy images.

Theoretical modelling of pitch: Because the capillary number $C_{a} \ll 1$, the meniscus evolution is quasi-static. Thus, at any given instant the meniscus adopts its static shape, as shown in Figure 5a. We non-dimensionalize all lengths by the capillary length $l_{c}$. Thus, we define the non-dimensional variables: $\tilde{z}=z / l_{c}, \tilde{y}=y / l_{c}, \tilde{L}=L / l_{c}$, as well as a nondimensional time $\tilde{t}=t V / l_{c}$. Unless otherwise noted, in the following equations we use non-dimensional variables, even if the tildes are omitted to simplify the writing. The equation describing this shape is:[54] 
$y=C+\operatorname{arccosh}\left(\frac{2}{L-z}\right)-2\left(1-\frac{(L-z)^{2}}{4}\right)^{1 / 2}$

where $C$ is an integration constant obtained from the boundary condition $y=0$ at $z=0$ :

$C=2\left(1-\frac{L^{2}}{4}\right)^{1 / 2}-\operatorname{arccosh}\left(\frac{2}{L}\right)$

Meniscus break-up occurs when the minimum value of $y$ equals the height of the particle deposit, $h_{\mathrm{p}}$ (Figure $5 \mathrm{~b}$ ). The location of the minimum value of $y$ corresponds to $d y / d z$ $=0$ with $y$ defined by Equation (4). If $L<2$, the location of the minimum is $z_{\min }=L-\sqrt{2}$. The corresponding value of $y=y_{\min }$ is:

$y_{\min }=2\left(1-\frac{L^{2}}{4}\right)^{1 / 2}-\operatorname{arccosh}\left(\frac{2}{L}\right)+\operatorname{arccosh}(\sqrt{2})-\sqrt{2}$

(6)

The break-up condition is $y_{\min }=-h_{P}$. We suppose that the height of the deposit is proportional to the amount of particles deposited in the meniscus by evaporative transport. In dimensional terms,

$h_{P}=k c V_{e} t$

where $k$ is a constant relating the height of the deposit with its total particle mass. Rewriting Equation(10) in non-dimensional terms and combining it with equation (9) yields

$k c \frac{V_{e}}{V}\left(L_{b}-L_{0}\right)=-\left[2\left(1-\frac{L_{b}^{2}}{4}\right)^{1 / 2}-\operatorname{arccosh}\left(\frac{2}{L_{b}}\right)+\right.$

$\operatorname{arccosh}(\sqrt{2})-\sqrt{2}]$

Equation (8) allows calculating the meniscus height at breakup, $L_{b}$, as a function of $V$ and $c$. Here $L_{\mathrm{o}}$ is the initial height of the meniscus, corresponding to $h_{\mathrm{p}}=\mathrm{o}$. It is given by the equation

$\frac{\left(1-L_{0}^{2}\right)}{L_{0} \sqrt{4-L_{0}^{2}}}=\tan \theta_{c}$

The value of the contact angle $\theta_{\mathrm{c}}$ is related to the width-tothickness ratio $r$ of the final drop that sets the shape of the nanohelix deposit. The relationship is:

$r=\frac{1-\cos \left(\theta_{c} / 2\right)}{\sin \left(\theta_{c} / 2\right)} \approx \theta_{c}$

By fitting the experimental data in Figure $7(\mathrm{~b})$, we deduce $\theta_{c}=1^{\circ}$, for which Equation (9) yields $L_{0}=1.40$, which is the value used to obtain the theoretical prediction in Figure $7(\mathrm{a})$.

\section{ASSOCIATED CONTENT}

\section{Supporting Information.}

SI-1: Silica helix surface charge. SI-2: Dispersion of the silica nanohelices in the presence of polymer additives. SI-3: Detailed image treatment and approximations for the density deposition in the slip and the stick regions. SI-4: Detailed image treatment of the order parameter calculation. SI-5: Dip coating deposition of $30 \mathrm{~nm}$ diameter $\mathrm{SiO}_{2}$ spherical particles. SI-6: SEM images of silica helix at $30000 \mu \mathrm{m} / \mathrm{s}$, to understand the bands formed in the advection regime. SI-7: Helix rotation in a Jeffery orbit during advection.

\section{AUTHOR INFORMATION}

\section{Corresponding Author}

* e.pouget@cbmn.u-bordeaux.fr; Glenna.drisko@icmcb.cnrs.fr

\section{Author Contributions}

J.G. produced all materials. S.S. and D.M. performed electron microscopy characterization. J.H. performed AFM characterization. Y.B. and D.G.-R. developed theoretical models. J.G., R.O., D. G.-R., G.L.D. and E.P. co-wrote the article. R.O. and E.P. financed the research. The manuscript was written through contributions of all authors. All authors have given approval to the final version of the manuscript.

\section{Funding Sources}

JG is supported by the Chinese Scholarship Council. JG, SS and GLD are supported by the LabEx AMADEus (ANR-10LABX-42) in the framework of IdEx Bordeaux (ANR-10-IDEX03-02), i.e. the Investissements d'Avenir program of the French government managed by the Agence Nationale de la Recherche.

\section{Notes}

Any additional relevant notes should be placed here.

\section{ACKNOWLEDGMENT}

The authors thank Matthias Pauly for the development of Image J plugins. FEGSEM instrumentation was facilitated by the PLACAMAT in Bordeaux and the Institut des Matériaux de Paris Centre (IMPC FR2482) and was cofunded by Sorbonne Université, CNRS and by the C'Nano projects of the Région Ile-de-France. The authors are grateful to Jacques Leng for fruitful discussions.

\section{REFERENCES}

[1] Glotzer, S.C.; Some Assembly Required. Science 2004, 306, 419-420

[2] Glotzer, S.C.; Solomon, M.J.; Anisotropy of building blocks and their assembly into complex structures. Nature Materials 2007, 6, 557-562

[3] Velev, O.D.; Gupta, S.; Materials Fabricated by Micro- and Nanoparticles Assembly - The Challenging Path from Science to Engineering. Adv. Mater. 2009, 21, 1897-1905

[4] Chung, W. J.; Oh, J. W.; Kwak, K. W.; Lee, B. Y.; Meyer, J.; et al.; Biomimetic self-templating supramolecular structures. Nature 2011, 478,364

[5] Xu, L.; W., Ma; L., Wang; C., Xu; H., Kuang; N. A., Kotov ; Nanoparticle assemblies: dimensional transformation of nanomaterials and scalability. Chem. Soc. Rev. 2013, 42,3114--3126

[6] Paul, D.R. ; Robeson, L.M. ; Polymer nanotechnology : Nanocomposites. Polymer 20o8, 49 3187-3204

[7] Kumar, S.K.; Krishnamoorti , R.; Nanocomposites: Structure, Phase Behavior, and Properties. Annual Review of Chemical and Biomolecular Engineering 2010, 1, 37-58

[8] Decher G. et al, US Pat., WO2015173432 A1, 2015 
[9] Sekar, S. ; Lemaire, V. ; Hu, H. ; Decher, G. ; Pauly, M. ; Anisotropic optical and conductive properties of oriented $1 \mathrm{D}$-nanoparticle thin films made by spray-assisted self-assembly. Faraday Discussions 2016, 191, 373-389

[10] Hu, H.; Pauly, M.; Felix, O.; Decher, G.; Spray-assisted alignment of Layer-by-Layer assembled silver nanowires: a general approach for the preparation of highly anisotropic nano-composite films. Nanoscale 2017, 9 (3), 1307-1314

[11] Angly, J.; Iazzolino, A.; Slamon, J.-B.; Leng, J.; Chandran, S. P.; et al.; Microfluidic-Induced Growth and Shape-Up of ThreeDimensional Extended Arrays of Densely Packed Nanoparticles. ACS Nano 2013, 7, 6465

[12] Li, P.; Li, Y.; Zhou, Z.-K., Tang, S. ; Yu, X.-F. ; et al.; Evaporative Self-Assembly of Gold Nanorods into Macroscopic 3D Plasmonic Superlattice Arrays. Adv. Mater. 2016, 28, 2511-2517

[13 Han, W.; Lin, Z.; Learning from "Coffee Rings": Ordered Structures Enabled by Controlled Evaporative Self-Assembly. Angew. Chem. Int. Ed. 2012, 51, 1534-1546

[14] Huang, J.; Fan, R.; Connor, S.; Yang, P.; One-Step Patterning of Aligned Nanowire Arrays by ProgrammedDip Coating. Angew. Chem. Int. Ed. 2007, 46, 2414-2417

[15] Dugyala, V. R. ; Basavaraj, M. G. ; Self-assembly of nanoellypsoids into ordered structures via vertical deposition. RSC Adv. 2015,5, 60079-60084

[16] Feng, J.; Xia, H.; You, F.; Mao, H.; Ma, X.; et al.; Alignment of Ag nanowires on glass sheet by dip-coating technique. J. Alloys and Compounds 2018, 735, 607-612

[17] Kim, K. T.; Park, C.; Kim, C.; Winnik, M. A.; Manners, I.; Self-assembly of dendron-helical polypeptide copolymers: organogels and lyotropic liquid crystals. Chem. Commun. 2oo6, 1372-1374

[18] Dierking, I.; Al-Zangana, S.; Lyotropic Liquid Crystal Phases from Anisotropic Nanomaterials. Nanomaterials 2017, $7(10) 305$

[19] Anderson, V.J.; Lekkerkerker, H. N. W.; Insights into phase transition kinetics from colloid science, Nature 2002, 416, 811-815

[20] Srikantharajah, R.; Schindler, T.; Landwehr, I.; Romeis, S.; Unruh, T.; Peukert, W.; From evaporation-induced self-assembly to shear-induced alignment. Nanoscale 2o16, 8, 19882-19893

[21] Wargacki S. P.; Pate B.; Vaia, R.A.; Fabrication of 2D Ordered Films of Tobacco Mosaic Virus (TMV): Processing Morphology Correlations for Convective Assembly. Langmuir 2oo8, 24, 5439-5444

[22] Kuncicky, D. M.; Naik, R. R.; Velev, O. D.; Rapid Deposition and Long-Range Alignment of Nanocoatings and Arrays of Electrically Conductive Wires from Tobacco Mosaic Virus. Small 2006, 2, 1462

[23] Lu, Y.; Ganguli, R.; Drewien, C. A.; Anderson M. T.; Brinker, C. J.; et al.; Continuous formation of supported cubic and hexagonal mesoporous films by sol-gel dip-coating. Nature 1997, 389, $364-368$

[24] Jokinen, M.; Pätsi, M.; Rahiala, H.; Peltola, T.; Ritala, M.; Rosenholm, J. B. Influence of sol and surface properties on in vitro bioactivity of sol-gel-derived $\mathrm{TiO}_{2}$ and $\mathrm{TiO}_{2}-\mathrm{SiO}_{2}$ films deposited by dip coating method. Journal of Biomedical Materials Research 1998,42,295-302

[25] Schunk, P. R.; Hurd, A. J.; Brinker, C. J.. Free-meniscus coating processes. In Liquid film coating (pp. 673-708), 1997, Springer, Dordrecht

[26] Dimitrov, A. S.; Nagayama, K.. Continuous Convective Assembling of Fine Particles into Two-Dimensional Arrays on Solid Surfaces Langmuir 1996, 12, 1303

[27] Denkov, N. D.; Velev, O. D.; Kralchevsky, P. A.; Ivanov, I. B.; Yoshimura, H.; Nagayama, K.. Mechanism of formation of twodimensional crystals from latex particles on substrates. Langmuir 1992, 8, 3183
[28] Prevo, B.; Velev, O.; Materials Deposition in Evaporating Menisci-Fundamentals and Engineering Applications of the Convective Assembly Process. In Evaporative Self-Assembly Of Ordered Complex Structures (pp. 109-155), 2012, World Scientific

[29] Huang, J.; Fan, R.; Connor, S.; Yang, P.. .One-Step Patterning of Aligned Nanowire Arrays by Programmed Dip Coating. Angew. Chem. Int. Ed. 2007, 46, 2414

[30] Farcau, C.; Moreira, H.; Viallet, B.; Grisolia, J.; Ressier, L.; Tunable conductive nanoparticle wire arrays fabricated by convective self-assembly on nonpatterned substrates. ACS Nano 2010, 4(12), 7275-7282

[31] Shimoda, H.; Oh, S. J.; Geng, H. Z.; Walker, R. J.; Zhang, X. B.; McNeil, L. E.; Zhou, O.; Self-assembly of carbon nanotubes. Adv. Mater. 2002, 14(12), 899-901

[32] Oda, R; Huc, I.; Schmutz, M.; Candau, S. J.; MacKintoshet, F. C.. Tuning bilayer twist using chiral counterions. Nature 1999, 399, 566-569

[33] Delclos, T.; Aimé, C.; Pouget, E.; Brizard, A.; Huc, I.; Delville, M.-H.; Oda, R., Individualized silica nanohelices and nanotubes: Tuning inorganic nanostructures using lipidic self-assemblies. Nano Lett. 2008, 8, 1929-1935

[34] Okazaki Y.; Buffeteau, T.; Siudyban, E.; Talaga, D.; Ryu, N.; Yagi, R.; Pouget, E.; Takafuji, M.; Ihara, H.; Oda, R.. Direct Observation of Siloxane Chirality on Twisted and Helical Nanometric Amorphous Silica, NanoLett. 2016, 16(10), 6411-6415

[35] Okazaki, Y.; Cheng, J.; Dedovets, D.; Kemper, G.; Delville, M.-H.; Durrieu, M.-C.; Ihara, H.; Takafuji, M.; Pouget, E.; Oda, R., Chiral colloids: Homogeneous suspension of individualized $\mathrm{SiO}_{2}$ helical and twisted nanoribbons. ACS Nano 2014, 8, 6863-6872

[36] Faustini, M.; Louis, B. ; Albouy, P. A. ; Kuemmel, M. ; Grosso, D., Preparation of Sol-Gel Films by Dip-Coating in Extreme Conditions. J. Phys. Chem. C 2o10, 114, 7637-7645

[37] Roland, S.; Gamys, C. G.; Grorenaud, J.; Boissé, S.; Pellerin, C., Prud'homme, R. E. ; Bazuin, C. G.. Solvent Influence on Thickness, Composition, and Morphology Variation with Dip-Coating Rate in Supramolecular PS- $b$-P4VP Thin Films. Macromolecules 2015, 48, 4823-4834

[38] Hofmann H. E.; Evaporation rates of organic liquids. Ind. Eng. Chem. 1932, 24(2): 135-140

[39] Le Berre M., Chen Y., Baigl D.. From convective assembly to landau-levich deposition of multilayered phospholipid films of controlled thickness. Langmuir 2009, 25(5):2554-2557

[40] Srikantharajah R., Schindler T., Landwer I., Romeis S., Unruh T., Peukert W.. From evaporation-induced self-assembly to shear-induced alignment. Nanoscale 2016, 8:19882

[41] Watanabe S., Inukai K., Mizuta S., Miyahara M. T., Mechanism for stripe pattern formation on hydrophilic surfaces by using convective self-assembly.

Langmuir 2009, 25, 7287

[42] Colosqui C.E.; Morris J. F.; Stone H.. Hydrodynamically driven colloidal assembly in dip coating. Phys. Rev. Lett. 2013, 110, 188302

[43] Jeffery J. B.. The motion of ellipsoidal particles immersed in a viscous fluid, Proc. Roy. Soc. A 1922, 102, 161-179

[44] Watanabe S.; Inukai K.; Mizuta S.; Miyahara M. T.. Mechanism for stripe pattern formation on hydrophylic surfaces by using convective self-assembly. Langmuir 2009, 25: 7287-7295

[45] Kaplan N.C.; Wu N.; Mandre S.; Aizenberg J.; Mahadevan L.. Dynamics of evaporative colloidal patterning. Phys. Fluids 2015, 27: 092105

[46] Leahy B. D., Koch D. L., Cohen I., Controlling the alignment of rodlike colloidal particles with time-dependent shear flows. J. Rheol. 2017, 61, 979

[47] Ghosh, M. ; Fan, F. ; Stebe, K. J.; Spontaneous pattern formation by dip coating of colloidal suspensions on homogeneous surfaces. Langmuir 2007, 23, 2180-2183 
[48] Becerril, H. A. ; Roberts, M. E. ; Liu, Z. ; Locklin, J. ; Bao, Z.; High-Performance Organic Thin-Film Transistors through Solution-Sheared Deposition of Small-Molecule Organic Semiconductor. Adv. Mater. 2008, 20, 2588-2594

[49] Huang J.; Kim F.; Tao A. R.; Connor S.; YangP.. Spontaneous formation of nanoparticle stripe patterns through dewetting. Nat. Mater. 2005, 4: 896-900

[50] Cazabat AM M.; Heslot F.; Troian S. M.; Carles P.. Fingering instability of thin spreading films driven by temperature gradients. Nature 1990, 346: 824-826

[51] Deblais A.; Harich R.; Colin A.; Kellay H.. Taming contact line instability for pattern formation. Nat. Commun. 2016 7, 12458

[52] Blell, R.; Lin, X.; Lindström, T.; Ankerfors, M.; Pauly, M.; Felix, O.; Decher, G.. Generating in-Plane Orientational Order in Multilayer Films Prepared by Spray-Assisted Layer-by-Layer Assembly. ACS Nano, 2017, 11, 84-94.

[53] Hu, H.; Pauly, M.; Felix, O.; Decher, G.. Spray-assisted alignment of Layer-by-Layer assembled silver nanowires: a general approach for the preparation of highly anisotropic nanocomposite films. Nanoscale, 2017, 9, 1307-21314.

[54] P.-G. de Gennes, F. Brochard-Wyart, D. Quéré, Capillarity and wetting phenomena. Drops, bubbles, pearls, waves. Springer, 2003.

[55] X. Lan, T. Liu, Z. Wang, A. O. Govorov, H. Yan, Y. Liu, DNA-Guided Plasmonic Helix with Switchqble Chirality. J. Am. Chem. Soc., 2018, 140, 11763-11770.

[56] Cheng, J.; Le Saux, G.; Gao, J.; Buffeteau, T.; Battie, Y.; Barois, P.; Ponsinet, V.; Delville, M.-H.; Ersen, O.; Pouget, E.; Oda, R. GoldHelix : Gold Nanoparticles forming 3D helical superstructures with controlled morphology and strong chiropitcal property. ACS Nano, 2017, 11, 3806-3818. 
Insert Table of Contents artwork here

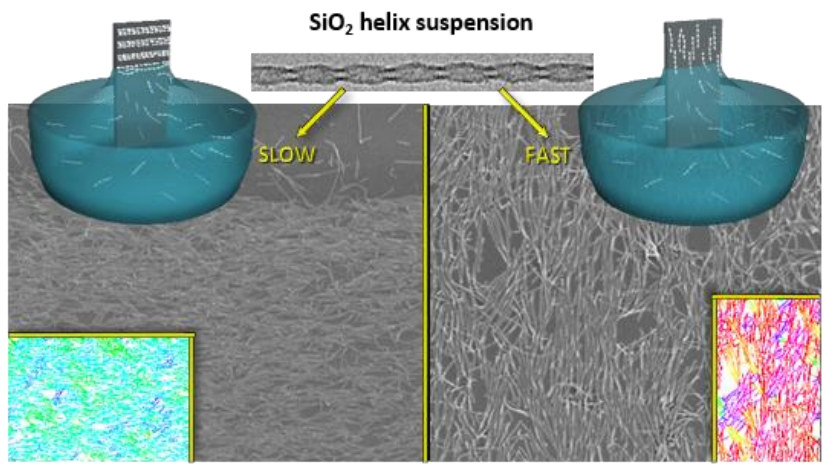

